\title{
Development and Evaluation of ELISA and qRT-PCR for Identification of Squash vein yellowing virus in Cucurbits
}

Craig G. Webster and William W. Turechek, United States Department of Agriculture-Agricultural Research Service (USDA-ARS), U.S. Horticultural Research Laboratory, Fort Pierce, FL 34945; Weimin Li, Citrus Research and Education Center, University of Florida, Lake Alfred 33850; and Biotechnology Research Institute, Chinese Academy of Agricultural Sciences, Beijing 100081; Chandrasekar S. Kousik, USDA-ARS, U.S. Vegetable Laboratory, Charleston, SC 29414; and Scott Adkins, USDA-ARS, U.S. Horticultural Research Laboratory, Fort Pierce, FL

\begin{abstract}
Squash vein yellowing virus (SqVYV) causes viral watermelon vine decline. To facilitate detection of SqVYV, enzyme linked-immunosorbent assay (ELISA) and quantitative reverse-transcription polymerase chain reaction (qRT-PCR) diagnostic methods were developed. Both methods were capable of detecting SqVYV in a wide range of cucurbit hosts. ELISA was able to detect virus in infected host tissue diluted to at least $1: 2,560$, which was sufficient for detection in symptomatic squash and watermelon plants. The qRT-PCR method was capable of reliably detecting as few as 3.4 copies of a cloned fragment of SqVYV genomic RNA with an average cycle threshold $\left(C_{t}\right)$ value of 36.4. The sensitivities and specificities for each detection method were estimated by latent class analysis for a set of inoculated squash and watermelon plants at two sampling scales. The scales were hierarchical, with individual

plants representing the upper scale and samples from the plant representing the lower scale. The number of samples per plant varied from 1 to 8 , and a plant was diagnosed positive if any of its samples tested positive. For all analyses, a cutoff $\mathrm{C}_{t}$ of 35 was chosen for qRT-PCR, which is approximately 2.5 cycles lower than the lowest $C_{t}$ value achieved for mock-inoculated plants (presumed to be a false positive). qRT-PCR showed high sensitivities $(\geq 0.99)$ at both sampling scales for squash and watermelon, whereas the sensitivities for ELISA ranged from 0.58 to 0.76 . The specificities for both tests were very similar ( $\geq 0.94$ ), with ELISA sometimes outperforming qRT-PCR. These diagnostic methods provide additional tools for the identification of SqVYV and management of SqVYV-induced watermelon vine decline.
\end{abstract}

Squash vein yellowing virus (SqVYV) is a whitefly-transmitted member of the genus Ipomovirus (Adkins et al. 2007; Li et al. 2008; Webb et al. 2012). Watermelon (Citrullus lanatus var. lanatus) is the most economically important host of SqVYV, in which it causes viral watermelon vine decline. SqVYV was first detected in watermelon in 2003 in Florida. The initial symptoms of infection in watermelon are a leaf wilt and petiole necrosis, which progress rapidly to a complete collapse of infected plants. Another characteristic symptom in watermelon fruit is the development of internal discoloration and necrosis in the rind and degradation of the flesh (Adkins et al. 2013). All other reported hosts of SqVYV are cucurbits, which typically display only mild vein yellowing symptoms on leaves and no fruit symptoms. These symptoms may be transient in appearance in some cucurbits (e.g., cucumber). Wilt and necrosis leading to plant death also occur in a limited number of other vining cucurbits (e.g., Cucurbita maxima) upon infection with SqVYV (Webster et al. 2013).

First found in Florida (Adkins et al. 2007), the known geographic range of SqVYV has increased with findings in Indiana, South Carolina, Georgia, California, Puerto Rico, Guatemala, and Israel (Acevedo et al. 2013; Batuman et al. 2015; Egel and Adkins 2007; Jeyaprakash et al. 2015; Reingold et al. 2016; Webster and Adkins 2012). Despite its fairly large geographical distribution, the genetic diversity of the virus is relatively low, with most Florida isolates

Corresponding author: S. Adkins; E-mail: scott.adkins@ars.usda.gov

Disclaimer: the use of trade, firm or corporation names in this publication is for the information and convenience of the reader. Such use does not constitute an official endorsement or approval by the United States Department of Agriculture or the Agricultural Research Service of any product or service to the exclusion of others that may be suitable.

Accepted for publication 14 September 2016.

This article is in the public domain and not copyrightable. It may be freely reprinted with customary crediting of the source. The American Phytopathological Society, 2017. and all Indiana, Georgia, and Puerto Rico isolates belonging to one predominant genotype (Webster and Adkins 2012). However, a second genotype has occasionally been detected in Florida (Webster and Adkins 2012), and only this genotype is reported from California, Guatemala, and Israel (Batuman et al. 2015; Jeyaprakash et al. 2015; Reingold et al. 2016). The sudden collapse and wilt of infected watermelon vines is consistently observed upon infection with both SqVYV genotypes. Collectively, this suggests that both SqVYV genotypes were recently introduced to the western hemisphere (Gilbertson et al. 2015; Webster and Adkins 2012).

For hosts such as watermelon and squash, experienced individuals can often correctly diagnose SqVYV infection based on characteristic symptoms such as the rapid progression of foliar wilt or necrosis and discoloration of the rind of watermelon, or vein yellowing in squash. Diagnosis based on rind necrosis is problematic because rind necrosis does not occur in all hosts and it requires destructive sampling of fruit (Webster et al. 2013). Visual diagnosis is further complicated by the frequent occurrence and coinfections with aphidtransmitted Papaya ringspot virus (PRSV) or whitefly-transmitted Cucurbit leaf crumple virus (CuLCrV) and Cucurbit yellow stunting disorder virus (CYSDV) that may be confused with or alter the symptoms of SqVYV infection (Turechek et al. 2010). Asymptomatic SqVYV infections are common in other cucurbit hosts and require laboratory methods to diagnose; for example, smell melon (Adkins et al. 2009), balsam apple (Adkins et al. 2008), or melon (Webster et al. 2013)].

Conventional reverse-transcription polymerase chain reaction (RT-PCR) (Adkins et al. 2008) and tissue-blot nucleic acid hybridization assays (TB) (Turechek et al. 2010) have both been developed for SqVYV detection. Although these methods are useful, there are some limitations. For instance, RT-PCR is currently the most accurate test for SqVYV (i.e., it is both sensitive and specific) but is impractical for large-scale testing of many samples due to its costs (Turechek et al. 2013). Conversely, TB can be used to test large numbers of samples but it is less sensitive and specific than RT-PCR. For some cucurbits, including bottle gourd (Lagenaria siceraria), TB is prone to producing false-positive results (Ling et al. 2013; Turechek et al. 2013; Webster et al. 2013). Both RT-PCR and TB are also capable of detecting both SqVYV genotypes. 
In this study, we developed an enzyme linked-immunosorbent assay (ELISA) as a cost-effective, high-throughput system for screening large numbers of samples, and a real-time or quantitative RT-PCR (qRT-PCR) as a highly sensitive method for more targeted surveys for diagnosis of SqVYV. Performances of both assays were quantified by latent class analysis (LCA) using data that represented an array of sample types to generate a wide range of possible test outcomes. Finally, we discuss the potential applicability of these methods to research applications and to management of viral watermelon vine decline.

\section{Materials and Methods}

Virus isolates. Initial development of diagnostic tests compared mock-inoculated and SqVYV-infected tissue from a variety of mature, fruit-bearing cucurbits collected in a previous study (Webster et al. 2013) to determine the likelihood of these tests to cross react with plant proteins (ELISA) or nucleic acids (qRT-PCR) of the various cucurbit species (Table 1). Florida isolates of commonly encountered cucurbit viruses Cucumber mosaic virus (CMV), CuLCrV, CYSDV, PRSV type W (PRSV-W), Tobacco streak virus (TSV), Watermelon leaf mottle virus (WLMV), Watermelon mosaic virus (WMV), Zucchini tigré mosaic virus (ZTMV), and Zucchini yellow mosaic virus (ZYMV) were kindly provided in infected cucurbit tissue by Susan Webb (Department of Entomology and Nematology, University of Florida) and used to determine the likelihood of these viruses to cross react with these SqVYV diagnostic reagents. In addition, 58 field samples of cucurbits were collected from southwest and west-central Florida during this study and used to qualitatively compare conventional and qRT-PCR.

As discussed in greater detail below, a series of experiments was used to further characterize qRT-PCR and ELISA test performances. A single isolate of the predominant Florida SqVYV genotype collected from watermelon in 2007 in Hendry County in southwest Florida (Webster and Adkins 2012) was used for these experiments. It was maintained in squash (C. pepo) 'Prelude II' (Seminis Seeds, Oxnard, CA) and watermelon 'Crimson Sweet' (Bunton Seed Co., Louisville, KY) by mechanical inoculation using $20 \mathrm{mM}$ sodium phosphate buffer ( $\mathrm{pH} 7.0$ ) containing $0.1 \%$ (wt/vol) sodium sulfite and $1 \%(\mathrm{wt} / \mathrm{vol})$ Celite. Plants were maintained in a temperaturecontrolled greenhouse under natural lighting with a daytime high temperature of $30^{\circ} \mathrm{C}$. Plants were drenched with the insecticide imidacloprid (Admire Pro; Bayer Crop Science, Research Triangle Park, NC) at 2 weeks postseeding and subsequently as needed to prevent whitefly infestation.

Development of ELISA for SqVYV detection. Prior attempts to develop useful antiserum from partially purified SqVYV virions and to use bacteria for expression of the SqVYV coat protein (CP) for use as an antigen were not successful, thus requiring exploration of alternative antigen sources. The SqVYV CP sequence was analyzed for unique amino acid sequences for synthesis of peptides to be used in antibody production. Two peptides were selected from within the SqVYV CP and synthesized (Biosynthesis, Lewisville, TX) for use as antigens. The first peptide $\left(\mathrm{NH}_{2}\right.$-AFDLAAKEAEIQKL RDEWDANKPSVTLSPF-OH) corresponds to SqVYV CP amino acids 1 to 30 (GenBank accession number EU259611) and was unique to SqVYV isolates. The second peptide $\left(\mathrm{NH}_{2}\right.$-EGEEDSDGE DEAKDKGKNVTTPDKKQILKG-OH) corresponds to SqVYV CP amino acids 67 to 96 and is also present in Cucumber vein yellowing virus (CVYV), the most closely related ipomovirus. Both peptides were highly conserved in SqVYV isolates, with the first peptide being identical and the second having at least $80 \%$ sequence identity to all known SqVYV isolates.

Individual male New Zealand white rabbits were injected with one or both of the synthetic peptides in an equal volume of Freund's Complete Adjuvant (Cocalico Biologicals, Reamstown, PA) using recommended protocols to produce three different polyclonal antisera. Booster injections were given to the rabbits with an equal volume of Freund's Incomplete Adjuvant subcutaneously or intramuscularly at 1 and 2 weeks. Test bleeds were made at 5 and 8 weeks, with an exsanguination bleed at 12 weeks.
All three antisera were tested in multiple formats. Only the antiserum produced from the mixture of the two synthetic peptides was useful, and only in an indirect plate-trapped antigen (PTA)-ELISA format. Briefly, tissue was homogenized in coating buffer $(15 \mathrm{mM}$ $\mathrm{Na}_{2} \mathrm{CO}_{3}, 35 \mathrm{mM} \mathrm{NaHCO}$, and $0.5 \mathrm{mM}$ polyvinyl pyrrolidone) at a ratio of 1:10 (wt/vol), with $100 \mu l$ of homogenate added per well to a polystyrene plate and incubated overnight at $4{ }^{\circ} \mathrm{C}$. The following day, primary antibody was diluted 1:250 (vol/vol) and $100 \mu \mathrm{l}$ was added and incubated at $37^{\circ} \mathrm{C}$ for $3 \mathrm{~h}$, followed by incubation with alkaline phosphatase conjugated goat-antirabbit antiserum (GAR-AP; Bio-Rad, Hercules, CA) diluted 1:3000 (vol/vol). Both primary and secondary antibodies were diluted in $1 \times$ phosphate-buffered salineTween (1× PBST; $140 \mathrm{mM} \mathrm{NaCl}, 1 \mathrm{mM} \mathrm{KH} \mathrm{PO}_{4}, 8 \mathrm{mM} \mathrm{Na}_{2} \mathrm{HPO}_{4}$, $2.5 \mathrm{mM} \mathrm{KCl}$, and $0.5 \%$ [vol/vol] Tween 20 [pH 7.4]) containing $0.5 \mathrm{mM}$ polyvinylpyrrolidone. Plates were washed three times with $1 \times$ PBST between all steps. Plates were developed $(100 \mu \mathrm{l}$ of $1 \mathrm{mg} / \mathrm{ml} p$-nitrophenyl phosphate [Sigma-Aldrich, St. Louis] dissolved in $0.01 \mathrm{M}$ diethanolamine buffer [pH 9.6]) and read using an MRX II plate reader (Dynex, Chantilly, VA), with readings twice that of mock-inoculated plants considered positive.

Development of qRT-PCR for SqVYV detection. A Taqman assay (Applied Biosystems, Foster City, CA) for real-time detection and quantification of the nuclear inclusion $b(\mathrm{NIb})$ gene of SqVYV was developed. Primers and probes were created to amplify a 133-bp region (nucleotides 7,095 to 7,227 of accession number EU259611) within the NIb gene in the $3^{\prime}$ portion of the SqVYV genome. Primers (SqVYV NIb-F, 5'-GTGAAGTCCAGAAGCAAG-3'; SqVYV NIb-R, 5'-ATGGAAGGTCCATACCTC-3') and probe (SqVYV NIb-P, 5'-FAM-ATCAAGGGCAAGCGAGCTTCTTTC-BHQ-3') were designed using an online development tool (PrimerQuest; Integrated DNA Technologies, Coralville, IA; www.idtdna.com) using default parameters and were synthesized by Biosearch Technologies (Novarto, CA).

Total RNA was extracted from $100 \mathrm{mg}$ of plant tissue using Trizol (Invitrogen, Carlsbad, CA). First-strand cDNA was synthesized using a degenerate potyvirus $3^{\prime}$ terminus primer (Gibbs and Mackenzie 1997), as previously described (Adkins et al. 2008). Reactions $(20 \mu \mathrm{l})$ were prepared in 96-well plates using $1 \mu \mathrm{l}$ of first-strand cDNA, Express $2 \times$ Fast master mix (Invitrogen), and primers and probe at final concentrations of 300 and $100 \mu \mathrm{M}$, respectively. The passive reference dye carboxy-X-rhodamine was added to a final

Table 1. Results of Squash vein yellowing virus (SqVYV) detection in mockinoculated and SqVYV-infected cucurbits

\begin{tabular}{|c|c|c|c|c|}
\hline \multirow[b]{2}{*}{ Species and cultivar } & \multicolumn{2}{|c|}{ Mock } & \multicolumn{2}{|c|}{ SqVYV } \\
\hline & $\overline{\text { ELISA }^{\mathbf{a}}}$ & $\overline{q R T-P C R}{ }^{b}$ & $\overline{\text { ELISA }^{a}}$ & qRT-PCR $^{b}$ \\
\hline $\begin{array}{l}\text { Citrullus lanatus } \\
\text { 'Crimson Sweet' }\end{array}$ & - & 38.4 & + & 18.3 \\
\hline $\begin{array}{l}\text { C. lanatus 'Black } \\
\text { Diamond' }\end{array}$ & - & $\geq 40$ & + & 18.1 \\
\hline C. lanatus 'Tri-X 313' & - & 37.8 & + & 20.1 \\
\hline $\begin{array}{l}\text { C. lanatus var. citroides } \\
\text { 'Ojakkyo' }\end{array}$ & - & $\geq 40$ & + & 21.6 \\
\hline $\begin{array}{l}\text { Cucurbita maxima } \\
\text { 'Buttercup Green' }\end{array}$ & - & nt & + & 20.6 \\
\hline C. реро 'Prelude II' & - & 37.6 & + & 19.9 \\
\hline C. pepo 'Zucchini Hybrid' & - & nt & + & 15.9 \\
\hline $\begin{array}{l}\text { Lagenaria siceraria } \\
\text { 'Birdhouse' }\end{array}$ & - & 39.7 & $\mathrm{nt}$ & 28.6 \\
\hline Momordica charantia & - & 38.6 & $\mathrm{nt}$ & 22.5 \\
\hline
\end{tabular}


synthesized peptides of SqVYV coat protein was tested for cross reactivity against a range of mock-inoculated and SqVYV-infected cucurbits. Values twice the background of the mock-inoculated samples were considered positive; $\mathrm{nt}=$ not tested.

${ }^{b}$ Average cycle threshold $\left(\mathrm{C}_{\mathrm{t}}\right)$ values of the quantitative reverse-transcription polymerase chain reaction (qRT-PCR) assay tested for cross reactivity against a range of mock-inoculated and SqVYV-infected cucurbits. Samples were tested in triplicate. 
concentration of $500 \mathrm{nM}$. All samples, standards, and controls were tested in duplicate or triplicate on an ABI Prism 7500 Fast thermocycler (Applied Biosystems), Eppendorf Mastercycler ep realplex ${ }^{4}$ (Eppendorf, New York), or Bio-Rad IQ5 Thermocycler (Bio-Rad) using a program of $95.0^{\circ} \mathrm{C}$ for $20 \mathrm{~s}$ followed by 40 cycles at $95^{\circ} \mathrm{C}$ for $3 \mathrm{~s}$ and $60^{\circ} \mathrm{C}$ for $30 \mathrm{~s}$, with data collection made in the $60^{\circ} \mathrm{C}$ for $30 \mathrm{~s}$ step.

Minimum detectable concentration and efficiency of qRTPCR. Segments of the SqVYV genome containing the region amplified by the qRT-PCR and conventional RT-PCR methods $(6,368$ to 7,963 and 8,493 to 9,563 nucleotides [nt], respectively, of accession number EU259611) were amplified using primers as previously described (Adkins et al. 2008; Li et al. 2008). Both amplicons were excised and purified (Qiagen, Valencia CA) from a $1 \%$ agarose gel, and eluted in $30 \mu \mathrm{l}$ of elution buffer and quantified on a NanoDrop spectrophotometer (NanoDrop Technologies, Wilmington, DE). The copy number of the PCR products was estimated at $3.4 \times 10^{11}$ cop$\mathrm{ies} / \mu \mathrm{l}(\mathrm{NIb})$ and $8.3 \times 10^{10}$ copies/ $\mu \mathrm{l}(\mathrm{CP})$ using the lengths $1,596 \mathrm{nt}$ for NIb and 1,071 nt for CP, Avogadro's number $\left(6.02 \times 10^{23}\right.$ copies/ mole), and average nucleotide weight $(660 \mathrm{~g} / \mathrm{mol})$ in the calculations. Ten-fold dilutions of each product were made from $10^{-4}$ to $10^{-10}$ (estimated at $3.4 \times 10^{7}$ to 340 copies $/ \mu 1$ for NIb and $8.3 \times 10^{6}$ to 8 copies $/ \mu l$ for $\mathrm{CP}$ ) and used to construct a standard curve to estimate the efficiency of the assay. Standards containing from $3.4 \times 10^{3}$ to $3.4 \times 10^{8}$ copies/ reaction were run on each plate of samples and were used to estimate the viral RNA concentration from cycle threshold $\left(C_{t}\right)$ values for unknown samples.

Construction of datasets and statistical analysis. Appropriate analysis of a diagnostic tool requires data containing the likely range of possible outcomes collected over a diverse set of conditions. Data should consist of a population of samples that have been exposed to the pathogen, typically referred to as "cases", and another population that has not, known as the "controls". Using these as guidelines, four sets of data were used to evaluate the ELISA and qRT-PCR tests for SqVYV. All plants discussed below were mechanically inoculated on a single, mature leaf, as described above. In addition, samples were also tested for SqVYV by TB and conventional RT-PCR, and through simple visual assessment for comparative and analytical purposes, also described below (Adkins et al. 2009; Turechek et al. 2010).

The first dataset was constructed to account for differences that might result from dissimilarities in the spatial distribution and accumulation of virus within plants (Turechek et al. 2010). Eight samples were collected along the length of each plant: the petiole and leaf tissue (squash) or vine segment (watermelon) from the inoculated leaf; the leaf immediately above the inoculated leaf; the youngest, fully expanded leaf; the crown tissue; and the growing tip. Each sample was first visually rated for symptoms of SqVYV infection. Samples were then processed and tested by TB and ELISA, and RNA was extracted for subsequent testing by RT-PCR within a few days of collection. The remaining cDNA was stored frozen until tested by qRTPCR once this assay was developed, approximately 3 years later. In total, 64 samples (8 each from eight plants) were tested.

The second dataset was collected from 110 plants ( 55 watermelon and 55 squash) and was constructed to account for differences that might result from dissimilarities in the temporal distribution and accumulation of virus within plants from inoculation to 14 days postinoculation (dpi). However, 15 of the 55 squash plants were used in a preliminary study. At 0 and $1 \mathrm{dpi}$, the petiole of the inoculated leaf (squash) or the crown (watermelon) were rated for symptoms of SqVYV and then tested for SqVYV by TB, ELISA, RT-PCR, and qRT-PCR as above. At 4, 5, 6, 7, 8, and 14 dpi, two samples per plant-petioles of the inoculated and youngest leaves (squash) or crown and growing tip (watermelon)-were collected, rated, and tested for SqVYV as above.

The third dataset consisted of 26 PRSV-inoculated watermelon plants and 7 PRSV-inoculated squash plants. PRSV commonly infects both watermelon and squash in Florida, and testing of this group of plants was done to measure the effect of PRSV infection, if any, on test performance. Plant samples were first visually examined for symptoms that can be confused as SqVYV and then tested immediately for SqVYV by TB, ELISA, RT-PCR, and qRT-PCR (i.e., cDNA was not stored frozen). For each watermelon plant, the crown, a segment from a noninoculated vine, and the growing tip of the plant were tested. On each squash plant, a petiole segment from the mockinoculated and the newest and second noninoculated leaves were tested. In total, 99 samples from 33 plants were tested.

The final dataset consisted of 18 mock-inoculated squash plants. Plant samples were rated for symptoms that can be confused as SqVYV and then tested immediately for SqVYV by TB, ELISA, RT-PCR, and qRT-PCR. On each squash plant, a petiole segment from the mock-inoculated and the newest and second noninoculated leaves were tested. In total, 54 samples from 18 plants were tested.

LCA. Sensitivities, specificities, and positive predictive values for the five SqVYV diagnostic tests were estimated at two sampling scales using LCA: the individual sample scale and the entire plant scale (Turechek et al. 2013). The sensitivity of a test is defined as the conditional probability of a positive test result given that the test subject is infected, the specificity is the conditional probability of a negative test result given that the subject is not infected, and the positive predictive value is the probability of infection for a given set of test results.

In a latent class model, it is assumed that the responses of the manifest variables (i.e., test results) are the result of an unobserved (i.e., latent) structure. In our case, the latent structure is the disease status of the samples or plants. LCA further requires that the manifest variables be categorical variables. Thus, qPCR results were converted to binary values by labeling samples with $C_{t} \leq 35$ as positive for SqVYV; otherwise, the sample was considered negative. With $d=5$ diagnostic tests, each giving two possible results, there are $2^{d}=32$ possible response patterns (Table 2 ).

In the diagnostic setting, the latent class methodology takes advantage of the cross-classified test results and uses a maximumlikelihood approach to assign individuals into one of two mutually exclusive latent classes (infected and noninfected) to estimate the individual test sensitivities and specificities. At the sample scale, test characteristics were determined directly from the test results of the individual samples. At the plant scale, a plant was labeled positive for SqVYV if any of the sample-scale samples originating from that plant tested positive; otherwise, the plant was considered free of infection.

A fuller account of LCA can be found in Turechek et al. (2013). We used PROC LCA in SAS (version 9.3; SAS Institute, Cary, $\mathrm{NC})$ to fit the latent class models by treating disease status as a two-class latent variable (Lanza et al. 2007, 2011). PROC LCA is an add-on procedure for SAS that can be obtained free of charge through Pennsylvania State University's Methodology Center's website (https://methodology.psu.edu/ra/lca).

\section{Results}

Development of ELISA for SqVYV detection. Antiserum background and specificity. Antiserum produced from the mixture of two synthetic peptides proved highly reliable for the detection of SqVYV in a range of host species when tested in an indirect PTA-ELISA format, with no significant cross reactivity observed with mockinoculated controls (Table 1). The test did not cross react with other common cucurbit viruses, including aphid-transmitted CMV, PRSV$\mathrm{W}, \mathrm{WMV}, \mathrm{ZTMV}$, and ZYMV; or whitefly-transmitted CuLCrV and CYSDV (data not shown). For regulatory reasons, we were not able to directly test ELISA on CVYV or the SqVYV genotype typical in California (Batuman et al. 2015), Guatemala (Jeyaprakash et al. 2015), or Israel (Reingold et al. 2016); however, based on alignments of the deduced CP amino acid sequences ( $>80 \%$ identity and minimum of 10 consecutive identical amino acids), our antibodies were predicted to detect this SqVYV genotype. In addition, we provided our SqVYV antiserum to researchers in California who directly demonstrated detection of their SqVYV isolate (Batuman et al. 2015).

Minimum detectable concentration. When infected plant material was homogenized, diluted, and tested by ELISA, the virus was consistently detectable in watermelon crown and squash leaf samples at dilutions of 1 part in 2,560 (absorbance at $405 \mathrm{~nm}\left[\mathrm{~A}_{405}\right]$ of 0.839 and 
0.393, respectively; Fig. 1). The virus was often detectable in watermelon crown samples at the next highest dilution of 1 part in 10,240 $\left(\mathrm{A}_{405}\right.$ of 0.410$)$, and greater absorbance values were observed for infected watermelon crown compared with squash leaf samples (Fig. 1). Absorbance values from mock-inoculated control plant tissue $\left(\mathrm{A}_{405}\right.$ of 0.122$)$ were similar to those of buffer-only controls $\left(\mathrm{A}_{405}\right.$ of 0.102), indicating a low level of background noise in the test.

Development of qRT-PCR for SqVYV detection. PCR efficiency, primer cross reactivity, and performance on field samples. The qRT-PCR assay was determined to be an efficient assay with a high linear correlation between the logarithm of virus concentration and $\mathrm{C}_{\mathrm{t}}$ value, as determined from the standard curve $\left(R^{2}=0.997\right.$; Fig. $2)$. Equivalent results were obtained with all three thermocyclers tested (data not shown) but the ABI 7500 machine was used for all experiments presented here. The efficiency of the reaction, as estimated from the slope of the standard curve, was 95.3\%. Additionally, an estimated 3.4 copies of the target region per reaction could be detected, which gave, on average, $\mathrm{a} \mathrm{C}_{\mathrm{t}}$ of 36.4 . This detection limit was approximately 2,440 times lower than the existing conventional RTPCR method (Adkins et al. 2008), which required an estimated 8,300 copies of the SqVYV-CP gene (data not shown).

SqVYV qRT-PCR was tested against the same common cucurbit viruses used in ELISA above and showed no amplification after 40 cycles, except for CYSDV, which gave a $C_{t}$ of 38.7. However, several mock-inoculated cucurbit species also showed amplification at $\mathrm{C}_{\mathrm{t}}>37.5$ (Table 1); therefore, the cross reaction seen with CYSDV may be partly associated with the cucurbit background rather than the virus. Low-level amplification from noninfected plant samples is common and is the reason why cutoff $\mathrm{C}_{\mathrm{t}}$ values are typically set at 35. This is approximately $2.5 \mathrm{C}_{\mathrm{t}}$ lower than the lowest reading in Table 1.

When tested on a collection of 58 cucurbit samples collected from southwest and west-central Florida, 20 samples tested positive by conventional RT-PCR; the remaining 38 tested negative. For qRTPCR, the range of $C_{t}$ values observed among the positive RT-PCR samples was 15.5 to 28.0 , whereas the range observed among the negative RT-PCR samples was 32.5 to 40.0 . Nearly all of the SqVYV-negative samples were coinfected with CuLCrV, CYSDV, or PRSV-W.

Estimation of the sensitivity and specificity of ELISA and qRT-PCR by LCA. Complete agreement among all five test results - either all positive or all negative - was found in approximately $57 \%$ of the sample-scale samples for both squash ([19 all positive +96 all negative $] / 198$ total) and watermelon ([10 all positive +109 all negative $] / 207$ total), and in $69 \%$ ([24 all positive +30 all negative $] / 78$ total) and $61 \%$ ([8 all positive +44 all negative $] / 85$ total) of the plant-scale samples for squash and watermelon, respectively (Table 2). There was a greater number of response patterns to consider at the sample scale than the plant scale, which is likely due to the greater number of samples at that scale.

At both the sample and plant scales, the sensitivities and specificities of qRT-PCR and RT-PCR were greater than 0.95 and often greater than 0.97 (Table 3), as expected. For the other three tests, the presence of typical virus symptoms was the least sensitive diagnostic, except on squash at the plant scale, where both ELISA and TB had lower sensitivity. This can be attributed to the highly characteristic vein yellowing that develops with SqVYV-infected squash plants, along with the skill of the raters and, perhaps, knowing that plants were inoculated. ELISA tended to be more sensitive than TB, except on watermelon at the plant scale. The specificities were all greater than 0.91 for the remaining tests, except for TB in watermelon (Table 3 ). The positive predictive values were greater than 0.95 when two or more tests gave positive results, as long as one of those tests was qRT-PCR (Table 4). The only exception was with $\mathrm{TB}$, where the positive predictive values were 0.79 and 0.61 for squash and watermelon, respectively, at the sample scale.

\section{Discussion}

ELISA and qRT-PCR methods for SqVYV detection were developed and validated by determining minimum detectable concentrations, the likelihood to cross react with other common cucurbit viruses or cucurbit host tissue, and through testing of a set of field

Table 2. Observed test response patterns of five diagnostic tests for Squash vein yellowing virus and the number of squash and watermelon samples testing positive $(+)$ or negative $(-)$ for each pattern at two sampling scales

\begin{tabular}{|c|c|c|c|c|c|c|c|c|}
\hline \multicolumn{5}{|c|}{ Test response patterna } & \multicolumn{2}{|c|}{ Sample scale } & \multicolumn{2}{|c|}{ Plant scale ${ }^{b}$} \\
\hline Symp & ELISA & TB & RT-PCR & qRT-PCR & Squash & Watermelon & Squash & Watermelon \\
\hline+ & + & + & + & + & 19 & 10 & 24 & 8 \\
\hline+ & + & + & - & + & 1 & - & - & - \\
\hline+ & + & - & + & + & 14 & 8 & 9 & - \\
\hline+ & - & + & + & + & 1 & 5 & - & 3 \\
\hline+ & - & + & - & + & - & - & 1 & - \\
\hline+ & - & + & - & - & 2 & - & 1 & - \\
\hline+ & - & - & + & + & 6 & 3 & 4 & - \\
\hline- & + & + & + & + & 19 & 19 & - & 8 \\
\hline- & + & + & - & + & 1 & - & - & - \\
\hline- & + & + & - & - & - & 1 & - & 1 \\
\hline- & + & - & + & + & 6 & 13 & - & 2 \\
\hline- & + & - & - & + & 1 & 1 & - & - \\
\hline- & + & - & - & - & 3 & - & 2 & - \\
\hline- & - & + & + & + & 6 & 9 & - & 8 \\
\hline- & - & + & + & - & - & 1 & - & - \\
\hline- & - & + & - & + & 2 & 1 & - & 1 \\
\hline- & - & + & - & - & 7 & 14 & 2 & 9 \\
\hline- & - & - & + & + & 13 & 12 & 5 & 1 \\
\hline- & - & - & + & - & 1 & - & - & - \\
\hline- & - & - & - & + & 1 & 1 & - & - \\
\hline- & - & - & - & - & 96 & 109 & 30 & 44 \\
\hline$\ldots$ & $\ldots$ & $\ldots$ & $\ldots$ & $\ldots$ & $n=198$ & $n=207$ & $n=78$ & $n=85$ \\
\hline
\end{tabular}

a Symp = visual assessment of symptoms, ELISA = enzyme-linked immunosorbent assay, TB = tissue blot, RT-PCR = conventional RT-PCR, and qRT-PCR = quantitative RT-PCR. There are 32 possible test response patterns with five diagnostic tests each having two possible outcomes, but only those patterns where test results occurred are shown. For qRT-PCR, $\mathrm{C}_{\mathrm{t}}=35.0$ was the cutoff.

${ }^{b}$ At the sample scale, a sample was classified as positive (or negative) for SqVYV directly from the test result of the individual sample. At the plant scale, a plant was labeled positive for SqVYV if any of the samples originating from that plant tested positive; otherwise, the plant was considered free of infection. 
samples shown previously to contain SqVYV. Furthermore, test performances were quantified in a series of experiments designed to provide the data necessary to allow estimation of test sensitivity and specificity through LCA. qRT-PCR was shown to have superior sensitivity to ELISA, TB, RT-PCR, and symptoms for identification of infected material in a wide range of hosts (Table 3). However, its specificity (i.e., the ability to correctly identify virus-free samples) was similar to RT-PCR and ELISA and on par with a number of the other tests when $C_{t}=35.0$ was selected as the cutoff.
When available, ELISA is often a preferred diagnostic method because it is simple to perform, provides rapid test results, can be used to process large numbers of samples, and is relatively inexpensive. The ELISA developed was less sensitive than both of the RT-PCR methods but its specificity was 0.94 or greater in the applications tested here, meaning that false positives occurred infrequently. Thus, a positive reaction by ELISA is strong indication of SqVYV infection. The ELISA method has distinct advantages over the existing TB method for SqVYV detection. First and foremost, the higher sensitivity and specificity of ELISA over TB in combination with the

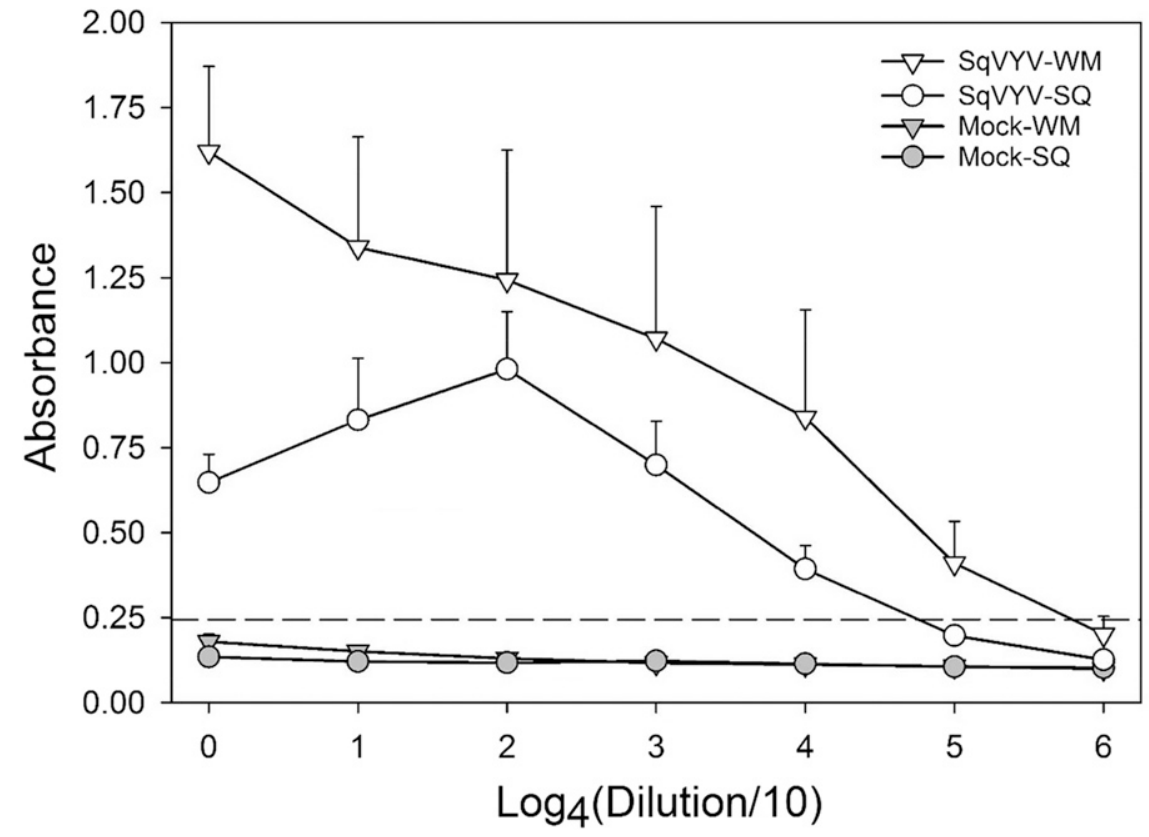

Fig. 1. Dilution series of Squash vein yellowing virus (SqVYV)-infected plant material with detection of the virus made by enzyme-linked immunosorbent assay (ELISA). Absorbance at $405 \mathrm{~nm}\left(\mathrm{~A}_{405}\right)$ of 1 in 10 (0), 1 in 40 (1), 1 in 160 (2), 1 in 640 (3), 1 in 2,560 (4), 1 in 10,240 (5), and 1 in 40,960 (6) dilutions (wt/vol) were made of 'Crimson Sweet' watermelon crown tissue (SqVYV-WM and Mock-WM) or 'Prelude II' squash leaf tissue (SqVYV-SQ and Mock-SQ) homogenized in coating buffer. Values represent the average and standard error (shown only in the positive direction for clarity in presentation) of nine replicate measurements from three independent ELISA plates. Dashed horizontal line of twice the average mock-inoculated plant reading indicates absorbance at which samples were considered positive.

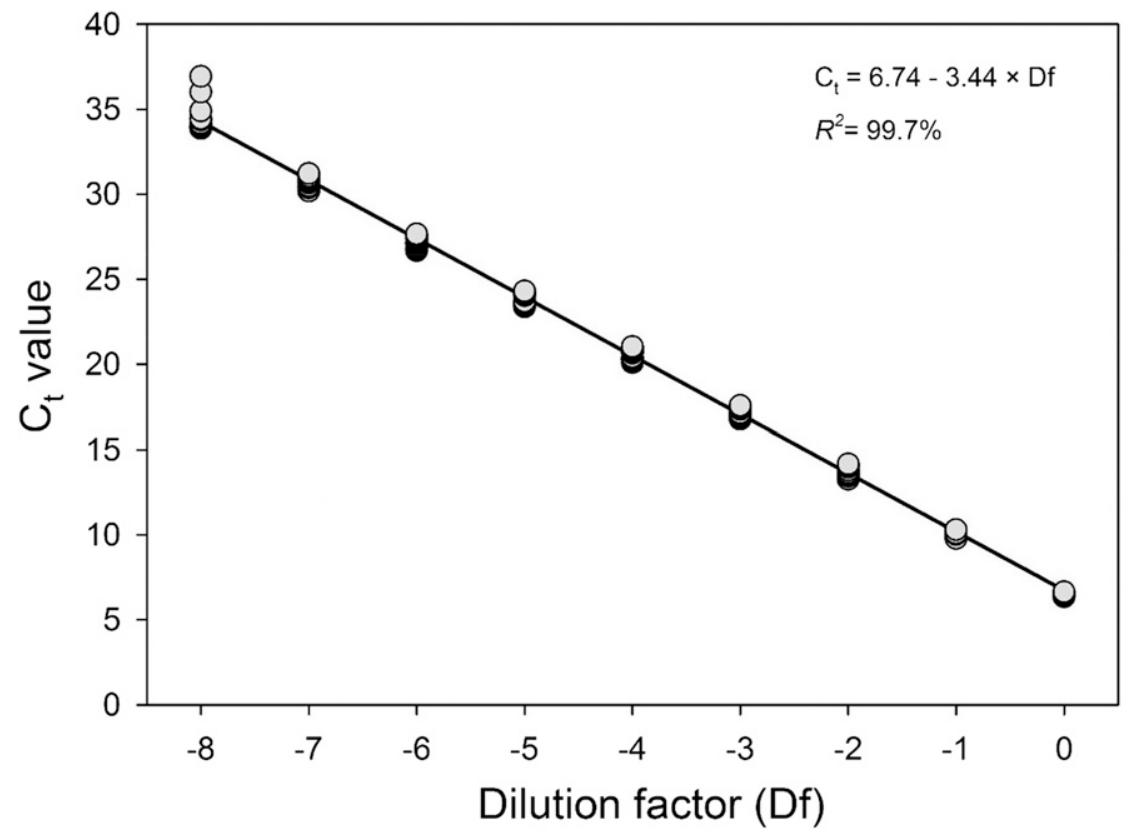

Fig. 2. Evaluation of amplification efficiency of a serial dilution of a Squash vein yellowing virus Nlb gene sequence amplicon by quantitative reverse-transcription polymerase chain reaction. The $\log 10^{-8}$ dilution was estimated to contain 3.4 copies of the target. The equation and fit of the standard curve are given. $C_{t}=$ cycle threshold. 
robust and simple interpretation of results, such as automated spectrophotometric plate reading, make the determination of infected samples with ELISA less subjective than TB. The TB method can be prone to difficulties in interpreting faint signals and nonspecific artifacts of blot development. For example, in previous studies, TB routinely produced a nonspecific signal from bottle gourd germplasm on blots (Ling et al. 2013; Turechek et al. 2013; Webster et al. 2013). In comparison, no nonspecific signal from bottle gourd was noted in ELISA testing in the current study (Table 1). Bottle gourd and other

Table 3. Estimates of the sensitivities, specificities, and standard errors (in parentheses) determined with a latent class analysis (LCA) model for five diagnostic tests of Squash vein yellowing virus (SqVYV) infection in squash and watermelon at the sample and plant scales ${ }^{\mathrm{a}}$

\begin{tabular}{|c|c|c|c|c|}
\hline \multirow[b]{2}{*}{ Test $^{\mathbf{b}}$} & \multicolumn{2}{|c|}{ Squash } & \multicolumn{2}{|c|}{ Watermelon } \\
\hline & Sen: & Spe & Se & ity \\
\hline \multicolumn{5}{|l|}{ Sample scale } \\
\hline Symptom & $0.464(0.053)$ & $0.981(0.013)$ & $0.318(0.052)$ & $0.999(0.002)$ \\
\hline ELISA & )) & & 0.624 & 0.99 \\
\hline TB & 3) & 0.916 & 0.543 & 0.87 \\
\hline RT-PCR & $0.952(0.025)$ & 0.989 & $0.975(0.021)$ & $0.996(0.009)$ \\
\hline qRT-PCR & $0.996(0.007)$ & $0.989(0.013)$ & $0.988(0.016)$ & $0.988(0.012)$ \\
\hline \multicolumn{5}{|l|}{ Plant scale } \\
\hline Symptom & $0.877(0.050)$ & $0.968(0.030)$ & $0.357(0.086)$ & $0.997(0.007)$ \\
\hline ELISA & $0.763(0.065)$ & $0.939(0.040)$ & $0.582(0.090)$ & $0.979(0.020)$ \\
\hline $\mathrm{TB}$ & .075) & $0.910(0.048)$ & $0.894(0.055)$ & $0.809(0.054)$ \\
\hline RT-PCR & 026) & 0.99 & $0.967(0.040)$ & 0.996 \\
\hline qRT-PCR & $0.994(0.013)$ & $0.993(0.014)$ & $0.991(0.018)$ & $0.991(0.019)$ \\
\hline \multicolumn{5}{|c|}{$\begin{array}{l}\text { a Estimated parameters were calculated with the add-on SAS procedure } \\
\text { PROC LCA. The probability of being in the SqVYV-infected latent class } \\
(=\text { disease incidence) was estimated to be } 0.444(0.04) \text { and } 0.3947(0.03) \\
\text { for squash and watermelon, respectively, at the sample scale; and } 0.551 \\
(0.06) \text { and } 0.362(0.05) \text { for squash and watermelon, respectively, at the plant } \\
\text { scale. For quantitative reverse-transcription polymerase chain reaction } \\
\text { (qRT-PCR), cycle threshold = } 35.0 \text { was the cutoff. } \\
\text { b ELISA }=\text { enzyme-linked immunosorbent assay and TB = tissue-blot nucleic } \\
\text { acid hybridization assay. }\end{array}$} \\
\hline
\end{tabular}

cucurbit species show resistance to SqVYV-induced vine decline and are of interest to breeding programs to introduce SqVYV resistance to watermelon germplasm (Kousik et al. 2009; Webster et al. 2013). Thus, it is essential for SqVYV diagnostic methods to function across a wide selection of cucurbit species.

The qRT-PCR method developed targeted the SqVYV NIb gene, making it independent of our other CP gene-based techniques. With a 40-cycle run, no detection of other cucurbit viruses commonly found in Florida was observed with the qRT-PCR tests. However, nonspecific reactions were occasionally seen with mock-inoculated cucurbits, manifested as high $C_{t}$ values (Table 1). In plant pathology, qPCR is generally used as a qualitative method (presence or absence), with the cutoff $C_{t}$ being chosen to reduce the likelihood of encountering such false positives as well as to provide a simple means to distinguish positive from negative samples (Chandelier et al. 2010). In our case, the cutoff $C_{t}$ was set to 35.0 , which is approximately 2.5 cycles lower than the lowest $C_{t}$ value achieved for the mock-inoculated plants shown in Table 1. Even with this low cutoff, the sensitivity of the test was still 0.99 at both the sample and plant scales for squash and watermelon. The cutoff $C_{t}$ of 35.0 was sufficient to allow SqVYV detection prior to symptom development in inoculated plants, when viral titer is presumably low, and allowed detection of approximately 3.4 copies of the viral RNA per reaction.

Comparing the performance of qRT-PCR to the RT-PCR method published for SqVYV (Adkins et al. 2008) on a collection of 58 field samples, there were six instances where qRT-PCR identified viral infection (with a cutoff $\mathrm{C}_{t}$ of 35) but RT-PCR did not. For these six tests, the $C_{t}$ values ranged between 32.5 and 34.7. At a minimum, this represents a 10 -fold difference in sensitivity based on the standard curve. Unfortunately, there were no field samples among the 58 with $\mathrm{C}_{\mathrm{t}}$ values between 28.0 (the maximum value among RT-PCRpositive samples) and 32.5 (the minimum value among RT-PCRnegative samples) to better compare the two sensitivities. Direct comparison of the two procedures is further complicated by the fact that the procedures target different regions of the SqVYV genome, as discussed above.

Dichotomizing continuous test results is a practice that is not necessarily recommended, largely because of the loss of information, the

Table 4. Positive predictive values estimated for the observed test response patterns of five diagnostic tests for Squash vein yellowing virus applied to squash and watermelon samples tested at two sampling scales

\begin{tabular}{|c|c|c|c|c|c|c|c|c|}
\hline \multicolumn{5}{|c|}{ Test response pattern ${ }^{\mathbf{a}}$} & \multicolumn{2}{|c|}{ Sample scale } & \multicolumn{2}{|c|}{ Plant scale } \\
\hline$\overline{\text { Symp }}$ & ELISA & TB & RT-PCR & qRT-PCR & Squash & Watermelon & Squash & Watermelon \\
\hline+ & + & + & + & + & 1.000 & 1.000 & 1.000 & 1.000 \\
\hline+ & + & + & - & + & 0.999 & - & 1.000 & - \\
\hline+ & + & - & + & + & 0.999 & 1.000 & - & - \\
\hline+ & - & + & + & + & 0.999 & 1.000 & - & 1.000 \\
\hline+ & - & + & - & + & - & - & 0.996 & - \\
\hline+ & - & + & - & - & 0.007 & - & 0.011 & - \\
\hline+ & - & - & + & + & 0.999 & 0.999 & 0.999 & - \\
\hline- & + & + & + & + & 0.999 & 1.000 & - & 0.999 \\
\hline- & + & + & - & + & 0.997 & - & - & - \\
\hline- & + & + & - & - & - & 0.041 & - & 0.014 \\
\hline- & + & - & + & + & 0.999 & 0.999 & - & 0.999 \\
\hline- & + & - & - & + & 0.957 & 0.971 & - & - \\
\hline- & + & - & - & - & 0.001 & - & 0.000 & - \\
\hline- & - & + & + & + & 0.999 & 0.999 & - & 0.999 \\
\hline- & - & + & + & - & - & 0.677 & - & - \\
\hline- & - & + & - & + & 0.793 & 0.610 & - & 0.713 \\
\hline- & - & + & - & - & 0.000 & 0.000 & 0.000 & 0.000 \\
\hline- & - & - & + & + & 0.998 & 0.999 & 0.997 & 0.998 \\
\hline- & - & - & + & - & 0.022 & - & - & - \\
\hline- & - & - & - & + & 0.230 & 0.159 & - & - \\
\hline- & - & - & - & - & 0.000 & 0.000 & 0.000 & 0.000 \\
\hline$\ldots$ & $\ldots$ & $\ldots$ & $\ldots$ & $\ldots$ & $n=198$ & $n=207$ & $n=78$ & $n=85$ \\
\hline
\end{tabular}

${ }^{\mathrm{a}}$ Symp = visual assessment of symptoms, ELISA = enzyme-linked immunosorbent assay, TB $=$ tissue blot, RT-PCR = conventional reverse-transcription polymerase chain reaction, and qRT-PCR $=$ quantitative RT-PCR. For qRT-PCR, cycle threshold $=35.0$ was the cutoff. There are 32 possible test response patterns, with five diagnostic tests each having two possible outcomes, but only those patterns where test results occurred are shown; $+=$ sample testing positive and $-=$ sample testing negative. 
increased likelihood of misclassification, and the loss of statistical power that occurs with artificial grouping (Dawson and Weiss 2012; Wang and Turechek 2016). Nonetheless, the practice is rampant for many reasons. Perhaps the most obvious is the desire for a yes or no answer, rather than a probability, and the fact that some common analyses (e.g., LCA) do not easily accommodate mixed data types (i.e., nominal, ordinal, binary, and so on). Numerous methods exist for cutoff selection and the topic is too large for discussion here (Caraguel et al. 2011; Chandelier et al. 2010). However, it should be noted that the reason that we did not endeavor to identify an "optimal" cutoff is that selection is largely based on the type of misclassifications one wishes to minimize. Those wishing to minimize false-positive classifications should establish lower values for the cutoff $C_{t}$, those wishing to minimize false-negative classifications should establish higher values for the cutoff $\mathrm{C}_{t}$, and those desiring a balance between the two can identify a cutoff based on one of several indices or graphical methods (Gönen 2007).

In the statistical analysis, it should be clear that the choice of the cutoff $\mathrm{C}_{t}$ will affect the estimates of qRT-PCR sensitivities and specificities; however, that choice also affects the estimates of the other tests because of how LCA uses cross-classified data (Wang and Turechek, 2016). For example, when a cutoff $C_{t}$ of 36.5 was chosen (rather than 35), the specificity of qRT-PCR at the sample scale for squash decreased from 0.989 to 0.964 . This, in turn, altered the sensitivities and specificities of the other tests, typically by less than 0.01 , and also lowered the positive predictive values for the five test results (data not shown).

The data were analyzed at two sampling scales that were hierarchical by design. At the plant scale, a plant was rated as positive if any of the plant's parts (= samples) tested positive. If the assumption is made that plant inoculation is $100 \%$ effective, then disease status at the plant scale was presumably known. However, for tests other than symptoms, confirmation of disease status depends on whether the plant sample being tested contains the pathogen. Because vascular-invading pathogens can be nonuniformly distributed in the plant, multiple samples from a plant are often analyzed to determine a plant's disease status. The largest differences in test performance at the two scales were found between the two tests that required subjective interpretation. For squash, the sensitivity of symptom expression at the sample scale was $41 \%$ lower than at the plant scale (Table 3). In general, it is simpler to identify the disease status of a plant when visualizing the entire plant rather than just a sample from that plant. Similarly, the sensitivity of TB was $35 \%$ lower at the sample scale than the plant scale for watermelon. This is likely a reflection of a combination of both the lower sensitivity of TB and the uneven distribution of virus in the plant. The specificity of most tests at both scales on both species was greater than 0.94 and often greater than 0.98 , with the exception of TB. Again, the propensity for false signal and subjective interpretation are the likely culprits in the reduction in test specificity.

In conclusion, two additional diagnostic methods were developed for identification of SqVYV. The advantages of ELISA are its user friendliness, quick turnaround of test results, and scalability to process large numbers of samples at a reasonably low cost. On the other hand, qRT-PCR can be multiplexed with tests for other common cucurbit-infecting viruses of Florida (e.g., CYSDV), as has been done for hosts commonly infected by several viruses (MortimerJones et al. 2009). Alternatively, the assay could be easily modified for detection of SqVYV within the whitefly vector, as was done for CVYV (Gil-Salas et al. 2007), which would be useful for monitoring viruliferous vector populations as a part of efforts to predict virus outbreaks. Both tests were shown to be accurate and robust and, thus, provide additional tools for the detection and management of SqVYV-induced watermelon vine decline.

The choice of which test to use will depend on the purpose (routine diagnostics or experimental), number of samples (few or many), and type of results desired (qualitative or quantitative). For routine SqVYV diagnostic purposes, we use conventional RT-PCR for small numbers of samples and ELISA for large numbers of samples. Conventional RT-PCR and ELISA both detect all known genotypes of SqVYV, as does TB. In contrast, qRT-PCR targets the lessconserved SqVYV NIb gene and is most useful for the predominant Florida SqVYV genotype. Accordingly, qRT-PCR offers a means to distinguish SqVYV genotypes without sequencing. A major use for qRT-PCR is in controlled experiments with the predominant Florida SqVYV genotype.

\section{Acknowledgments}

We thank S. Webb for providing cucurbit viruses for use in specificity testing; C. Vanderspool, B. Burns, and N. Miller for excellent technical assistance; and M. Hilf, K. Sims, and C. Latza for helpful advice and useful discussions on qRT-PCR. Financial support was provided, in part, by the United States Department of Agriculture Cooperative State Research, Education, and Extension Service Specialty Crop Research Initiative grant 2008-04890 and the National Watermelon Association.

\section{Literature Cited}

Acevedo, V., Rodrigues, J. C. V., de Jensen, C. E., Webster, C. G., Adkins, S., and Wessel-Beaver, L. 2013. First report of Squash vein yellowing virus affecting watermelon and bitter gourd in Puerto Rico. Plant Dis. 97:1516.

Adkins, S., McCollum, T. G., Albano, J. P., Kousik, C. S., Baker, C. A., Webster, C. G., Roberts, P. D., Webb, S. E., and Turechek, W. W. 2013. Physiological effects of Squash vein yellowing virus infection on watermelon. Plant Dis. 97:1137-1148

Adkins, S., Webb, S. E., Achor, D., Roberts, P. D., and Baker, C. A. 2007 Identification and characterization of a novel whitefly-transmitted member of the family Potyviridae isolated from cucurbits in Florida. Phytopathology 97: $145-154$

Adkins, S., Webb, S. E., Baker, C. A., and Kousik, C. S. 2008. Squash vein yellowing virus detection using nested polymerase chain reaction demonstrates that the cucurbit weed Momordica charantia is a reservoir host. Plant Dis. 92: $1119-1123$.

Adkins, S., Webster, C. G., Baker, C. A., Weaver, R., Rosskopf, E. N., and Turechek, W. W. 2009. Detection of three whitefly-transmitted viruses infecting the cucurbit weed Cucumis melo var. dudaim in Florida. Plant Health Prog. doi:10.1094/PHP-2009-1118-01-BR.

Batuman, O., Natwick, E. T., Wintermantel, W. M., Tian, T., McCreight, J. D., Hladky, L. L., and Gilbertson, R. L. 2015. First report of an ipomovirus infecting cucurbits in the Imperial Valley of California. Plant Dis. 99:1042.

Caraguel, C. G. B., Stryhn, H., Gagné, N., Dohoo, I. R., and Hammell, K. L. 2011. Selection of a cutoff value for real-time polymerase chain reaction results to fit a diagnostic purpose: Analytical and epidemiological approaches. J. Vet. Diagn. Invest. 23:2-15.

Chandelier, A., Planchon, V., and Oger, R. 2010. Determination of cycle cut off in real-time PCR for the detection of regulated plant pathogens. Bull. OEPP/EPPO Bull. 40:52-58.

Dawson, N. V., and Weiss, R. 2012. Dichotomizing continuous variables in statistical analysis: A practice to avoid. Med. Decis. Making 32:225-226.

Egel, D. S., and Adkins, S. 2007. Squash vein yellowing virus identified in watermelon (Citrullus lanatus) in Indiana. Plant Dis. 91:1056.

Gibbs, A., and Mackenzie, A. 1997. A primer pair for amplifying part of the genome of all potyvirids by RT-PCR. J. Virol. Methods 63:9-16.

Gilbertson, R. L., Batuman, O., Webster, C. G., and Adkins, S. 2015. Role of the insect supervectors Bemisia tabaci and Frankliniella occidentalis in the emergence and global spread of plant viruses. Annu. Rev. Virol. 2:67-93.

Gil-Salas, F. M., Morris, J., Colyer, A., Budge, G., Boonham, N., Cuadrado, I. M., and Janssen, D. 2007. Development of a real-time RT-PCR assay for the detection of Cucumber vein yellowing virus (CVYV) and Cucurbit yellow stunting disorder virus (CYSDV) in the whitefly vector Bemisia tabaci. J. Virol. Methods 146:45-51.

Gönen, M. 2007. Analyzing Receiver Operating Characteristic Curves with SAS. SAS Institute Inc., Cary, NC.

Jeyaprakash, A., Baker, C. A., Schubert, T. S., Badillo-Vargas, I. E., Roberts, P. D., Funderburk, J. E., and Adkins, S. 2015. First report of Squash vein yellowing virus in watermelon in Guatemala. Plant Health Prog. 16 113-114.

Kousik, C. S., Adkins, S., Turechek, W. W., and Roberts, P. D. 2009. Sources of resistance in U.S. plant introductions to watermelon vine decline caused by Squash vein yellowing virus. HortScience 44:1-7.

Lanza, S. T., Collins, L. M., Lemmon, D. R., and Schafer, J. L. 2007. PROC LCA: A SAS procedure for latent class analysis. Struct. Equation Model. 14:671-694.

Lanza, S. T., Dziak, J. J., Huang, L., Xu, S., and Collins, L. M. 2011. Proc LCA \& Proc LTA Users' Guide (Version 1.2.6). The Methodology Center, Pennsylvania State University, University Park. https://methodology.psu.edu

Li, W., Hilf, M. E., Webb, S. E., Baker, C. A., and Adkins, S. 2008. Presence of P1b and absence of HC-Pro in Squash vein yellowing virus suggests a general feature of the genus Ipomovirus in the family Potyviridae. Virus Res. $135: 213-219$. 
Ling, K.-S., Levi, A., Adkins, S., Kousik, C. S., Miller, G., Hassell, R. L., and Keinath, A. P. 2013. Development and field evaluation of multiple virusresistant bottle gourd (Lagenaria siceraria). Plant Dis. 97:1057-1062.

Mortimer-Jones, S. M., Jones, M. G. K., Jones, R. A. C., Thomson, G., and Dwyer, G. I. 2009. A single tube, quantitative real-time PCR assays that detects four potato viruses simultaneously. J. Virol. Methods 161:289-296.

Reingold, V., Lachman, O., Sela, N., Luria, N., and Dombrovsky, A. 2016. Watermelon fruit rot disease in Israel is caused by a distinct Squash vein yellowing virus (SqVYV) strain. Plant Dis. 100:1176-1183.

Turechek, W. W., Kousik, C. S., and Adkins, S. 2010. Distribution of four viruses in single and mixed infections within infected watermelon plants in Florida. Phytopathology 100:1194-1203.

Turechek, W. W., Webster, C. G., Duan, J., Roberts, P. D., Kousik, C. S., and Adkins, S. 2013. The use of latent class analysis to estimate the sensitivities and specificities of diagnostic tests for Squash vein yellowing virus in cucurbit species when there is no gold standard. Phytopathology 103:1243-1251.

Wang, H., and Turechek, W. W. 2016. A loop-mediated isothermal amplification assay and sample preparation procedure for sensitive detection of Xanthomonas fragariae in strawberry. PLoS One 11:e0147122.

Webb, S. E., Adkins, S., and Reitz, S. R. 2012. Semipersistent whitefly transmission of Squash vein yellowing virus, causal agent of viral watermelon vine decline. Plant Dis. 96:839-844.

Webster, C. G., and Adkins, S. 2012. Low genetic diversity of Squash vein yellowing virus in wild and cultivated cucurbits in the U.S. suggests a recent introduction. Virus Res. 163:520-527.

Webster, C. G., Kousik, C. S., Turechek, W. W., Webb, S. E., Roberts, P. D., and Adkins, S. 2013. Squash vein yellowing virus infection of vining cucurbits and the vine decline response. Plant Dis. 97:1149-1157. 